\title{
Smoothened Antagonist TAK-441
}

National Cancer Institute

\section{Source}

National Cancer Institute. Smoothened Antagonist TAK-441. NCI Thesaurus. Code C116874.

An orally bioavailable pyrrolopyridine derivative and Smoothened (Smo) antagonist with potential antineoplastic activity. Smo antagonist TAK-441 selectively binds to and inhibits the activity Smo, which is a cell surface co-receptor for ligands in the Hedgehog ( $\mathrm{Hh}$ ) family. This may result in a suppression of Hh-mediated signaling pathways, thereby inhibiting the growth of tumor cells in which this pathway is aberrantly activated. Smo is a G-protein coupled receptor that lies just downstream of the Hh cell surface receptor Patched-1 in the Hh pathway; in the absence of ligand, Patched-1 (Ptch1) inhibits Smo, and ligand binding to Ptch1 results in increased levels of Smo. The Hh-mediated signaling pathways play an important role in cellular growth and differentiation, and tissue repair; constitutive activation of this pathway is associated with uncontrolled cellular proliferation in a variety of cancers. 